\title{
Evolving Use of Androgen Deprivation Therapy in Prostate Cancer Management
}

Presented by James L. Mohler, MD

\section{Abstract}

Since its introduction more than 70 years ago, the use of androgen deprivation therapy (ADT) for prostate cancer has evolved to become part of a multimodal management approach. In this presentation from the NCCN 21st Annual Conference, James L. Mohler, MD, reviewed data that inform these strategies and gave his bottom-line recommendations on issues such as ADT plus radiotherapy, continuous versus intermittent ADT, ADT for positive nodes, and ADT plus docetaxel. He suggested that ADT plus radiation therapy should be used in patients at high risk, intermittent ADT is more appropriate for most patients than continuous ADT, and docetaxel should be given with ADT for high-volume metastatic disease.

J Natl Compr Cancer Netw 2016;14(5.5):663-665

The strategic application of androgen deprivation therapy (ADT) in the clinic is still evolving, according to James L. Mohler, MD, Chair of the Department of Urology and Associate Director of Translational Research, Roswell Park Cancer Institute, and Chair of the NCCN Guidelines Panel for Prostate Cancer. ADT includes numerous agents, alone or in combination with other drugs, and in a variety of strategies that patients and clinicians select together. At the NCCN 21st Annual Conference, Dr. Mohler described the evolution of ADT "from 1941 to the present" and offered his opinion on the clinical utility of various approaches.

\section{Estrogen: Try Parenteral Route}

Parenteral estrogen can be a useful agent in many patients whose disease has not responded to ADT, according to the 2013 PATCH trial, which involved patients with locally advanced disease. ${ }^{1}$ In that study, investigators found that patients responded equally as well to the estrogen patch as to a luteinizing hormone-releasing hormone (LHRH) agonist, and had good tolerability. The

Presented by James L. Mohler, MD, Department of Urology, Roswell Park Cancer Institute, Buffalo, New York.

Dr. Mohler has disclosed that he has no financial interests, arrangements, affiliations, or commercial interests with the manufacturers of any products discussed in this article or their competitors.

Correspondence: James L. Mohler, MD, Department of Urology, Roswell Park Cancer Institute, Elm and Carlton Streets, Buffalo, NY 14263.

E-mail: James.Mohler@RoswellPark.org study is currently continuing to its primary end point of overall survival (OS).

"We should think about trying estrogen parenterally. It has the same cancer control value, and seems safer," Dr. Mohler said.

\section{Optimal ADT}

LHRH agonists and antagonists have comparable utility, Dr. Mohler simply stated. "Antagonists are marketed as superior, but there are no data supporting this," he indicated.

Similarly, an LHRH agonist alone is as effective as an LHRH agonist plus an antiandrogen to achieve complete androgen blockade. In a study from 15 years ago, flutamide added to orchiectomy did not improve survival. ${ }^{2}$ Since that report, many randomized controlled trials and metaanalyses have shown little, if any, benefit to this approach, though many physicians still prescribe combined androgen blockade (CAB) with an LHRH agonist plus an antiandrogen.

"Adding an antiandrogen just increases side effects and costs; for that reason I am against it," he said.

\section{ADT Plus Radiation Therapy}

Does radiation therapy (RT) make ADT more effective, and does ADT improve RT? In the phase III EORTC 22991 trial involving 819 patients, ${ }^{3}$ the addition of short-term ADT to RT improved biochemical 
and clinical disease-free survival in patients with intermediate- and high-risk prostate cancer. Five-year biochemical disease-free survival was $82.6 \%$ in the combination group compared with $69.8 \%$ in the RT group (hazard ratio [HR], 0.52; $P<.001$ ).

Several subsequent trials also found benefit for the combined approach of ADT plus RT. The SPCG-7/SFUO-3 trial included 875 patients with locally advanced disease who received 3 months of CAB plus continuous flutamide or the same plus RT. Results showed a small OS advantage but a large reduction in biochemical recurrence. ${ }^{4}$ In $\mathrm{RTOG}$ 86-10, which evaluated RT after 3 months of ADT, the combination led to a reduction in the rates of prostate-specific antigen (PSA) failure, metastases, and cancer-specific mortality and showed a trend in improved OS. ${ }^{5}$

Results of the RTOG 94-08 trial found that ADT plus RT improved 10 -year OS (62\% vs $57 \%$, for RT alone; $P=.03$ ). However, a subanalysis by risk group showed improvements in overall and diseasespecific mortality primarily among patients with intermediate-risk disease, but no significant reductions among patients with low-risk disease. ${ }^{6}$

According to Dr. Mohler, ADT "shrinks the target, reduces side effects, and improves OS" and "absolutely" should be used with RT in patients at high risk. RT, however, may not be necessary for patients with favorable intermediate-risk disease, although it is an option in patients with unfavorable intermediate-risk disease. The RTOG is further evaluating ADT plus RT for intermediate-risk disease.

\section{ADT for Positive Nodes}

Should ADT be prescribed for patients found to have positive lymph nodes on prostatectomy? "Maybe," Dr. Mohler said.

An ECOG trial of early versus delayed ADT for node-positive disease found that early initiation of ADT improved OS $(P=.04)$, and reduced prostate cancer recurrence $(P<.0001)$ and biochemical failure $(P<.0001){ }^{7}$ These "unbelievable" results were not observed, however, in a propensity-matched analysis of the SEER-Medicare database. ${ }^{8}$

"The results from Messing et $\mathrm{al}^{7}$ were compelling, but no one has been able to reproduce them, and I doubt there will be another clinical trial of that exact population," he said. "With our improved staging, lymph node metastases are very rare in men undergoing prostatectomy. In my last 600 cases, we only had 6."

\section{Intermittent Versus Continuous ADT}

Dr. Mohler was clear on his position on continuous ADT. "I think no one should get it," he stated. "Continuous ADT is not informed by the data." His recommendation (strongly backed by data ${ }^{9-14}$ ) is to give induction ADT then intermittent ADT; this can be personalized according to end-of-induction PSA, he added.

The influential SWOG 9346 study showed a trend toward improved survival with continuous ADT: 5.8 versus 5.1 years (HR, 1.1; 95\% CI, 0.991.23). ${ }^{14}$ Differences in survival, if any, were driven by subsets with higher Gleason scores in nonmetastatic disease and by symptomatic bone metastases in the metastatic subset.

Although continuous ADT has been promoted based on study results, "It doesn't pass the 'man in the street test,"' according to Dr. Mohler. A recent meta-analysis of 6 randomized controlled trials showed that mortality was similar for intermittent versus continuous ADT, but quality of life was better, including erectile function, with intermittent ADT. ${ }^{13}$ Toxicities associated with ADT include fatigue, hot flashes, loss of libido, osteoporosis, and metabolic syndrome, and there is an increased risk for stroke, myocardial infarction, and diabetes that is associated with ADT. "Patients are up in arms about the side effects of continuous ADT," he commented. "Intermittent ADT is better for erectile dysfunction and quality of life."

Dr. Mohler recommended that treatment for metastatic disease should be guided by disease-related symptoms. In asymptomatic patients, he recommends ADT, and discussed the potential tradeoff between a potentially lesser survival benefit and better quality of life. For symptomatic patients, he considers continuous ADT; however, if PSA levels decrease to less than $4 \mathrm{ng} / \mathrm{mL}$ (and certainly $<0.2 \mathrm{ng} / \mathrm{mL}$ ), he believes intermittent ADT is preferable.

Dr. Mohler also advised clinicians to "rethink orchiectomy" as an alternative to ADT. A recent study found that orchiectomy was associated with a lower risk of fracture, peripheral arterial disease, and cardiac-related complications, and a similar risk of diabetes, thromboembolism, and cognitive decline. ${ }^{15}$ 


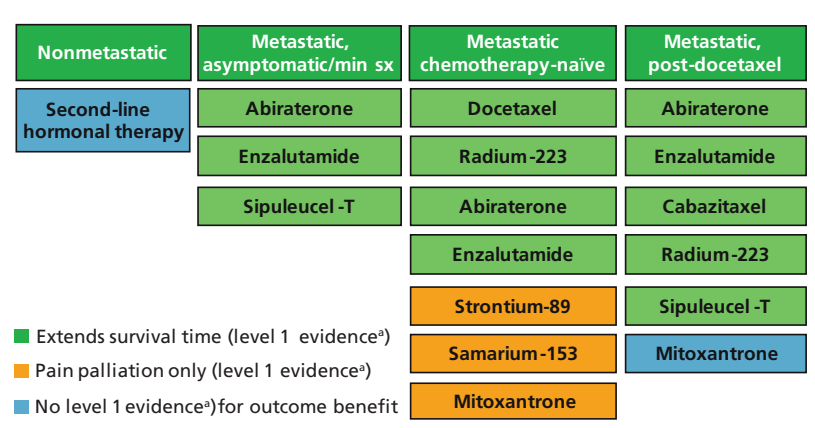

Figure 1. Overview of currently available main treatment options for castration-recurrent prostate cancer.

Abbreviations: CRPC, castration-recurrent or castration-resistant prostate cancer; $\mathrm{min} \mathrm{sx}$, minimally symptomatic.

aRecommendations based on the NCCN Guidelines for Prostate Cancer, Version 2.2016. Available at NCCN.org.

\section{ADT Plus Docetaxel}

Docetaxel can be added to ADT for high-volume disease (visceral metastases and/or $\geq 4$ bone metastases, including $\geq 1$ outside the pelvis or vertebral column), especially in otherwise healthy men, he said.

Sweeney et $\mathrm{al}^{16}$ recently reported a $40 \%$ reduction in risk of death with docetaxel plus ADT in men with high-volume disease $(P<.001)$, but found no significant difference in those with low-volume disease. Results from the recent STAMPEDE trial found median survival in patients to be 71 months with ADT alone and 81 months with the addition of docetaxel $(P=.001)$; the benefit was driven by the patient subset with bone metastases. ${ }^{17}$ Additionally, a recent meta-analysis showed the addition of docetaxel improved 4-year survival by an absolute $9 \%(\mathrm{HR}, 0.77 ; \mathrm{P}<.0001)$; the investigators concluded that this should be the standard of care when starting treatment for androgen-stimulated metastatic disease. ${ }^{18}$

"It makes sense that when the disease is found early, you treat it with chemotherapy in hopes of delaying progression, rather than allowing it to progress on ADT and then treating it with something the patient will tolerate more poorly," Dr. Mohler said.

\section{New Agents for ADT for Castration- Recurrent Prostate Cancer}

When men progress to castration-recurrent disease (CRPC), a number of new agents are now available, many of which offer a survival benefit (Figure 1).

"We have many different approaches that empower men; they have a smorgasbord to choose from, but at the same time it can confuse them. Even many physicians don't know when to use one and not another. How to sequence these agents and whether we can combine them is something we don't yet know," he said.

Cost is also an issue with these agents, he continued. Based on the treatment duration and benefit reported from clinical trials, Dr. Mohler concluded, "Patients can experience 23 months in survival after ADT fails, which will cost them and the health care system $\$ 532,000 "$ if all the treatments indicated for CRPC were used sequentially and each patient achieved the mean survival extension in the FDA registration trials.

\section{References}

1. Langley RE, Cafferty FH, Alhasso AA, et al. Cardiovascular outcomes in patients with locally advanced and metastatic prostate cancer treated with luteinising-hormone-releasing-hormone agonists or transdermal oestrogen: the randomised, phase 2 MRC PATCH trial (PR09). Lancet Oncol 2013;14:306316.

2. Eisenberger MA, Blumenstein BA, Crawford ED, et al. Bilateral orchiectomy with or without flutamide for metastatic prostate cancer. N Engl J Med 1998;339:1036-1042.

3. Bolla M, Gonzalez D, Warde P, et al. Improved survival in patients with locally advanced prostate cancer treated with radiotherapy and goserelin. N Engl J Med 1997;337:295-300.

4. Widmark A, Klepp O, Solberg A, et al. Endocrine treatment, with or without radiotherapy, in locally advanced prostate cancer (SPCG-7/SFUO-3): an open randomised phase III trial. Lancet 2009;373:301-308.

5. Roach M III, Bae K, Speight J, et al. Short-term neoadjuvant androgen deprivation therapy and external-beam radiotherapy for locally advanced prostate cancer: long-term results of RTOG 8610. J Clin Oncol 2008;26:585591.

6. Jones CU, Hunt D, McGowan DG, et al. Radiotherapy and short-term androgen deprivation for localized prostate cancer. N Engl J Med 2011;365:107-118.

7. Messing EM, Manola J, Sarosdy M, et al. Immediate hormonal therapy compared with observation after radical prostatectomy and pelvic lymphadenectomy in men with node-positive prostate cancer. N Engl J Med 1999;341:1781-1788.

8. Wong YN, Freedland S, Egleston B, et al. Role of androgen deprivation therapy for node-positive prostate cancer. J Clin Oncol 2009;27:100-105.

9. Crook JM, O'Callaghan CJ, Duncan G, et al. Intermittent androgen suppression for rising PSA level after radiotherapy. N Engl J Med 2012;367:895-903.

10. Higano CS. Intermittent versus continuous androgen deprivation therapy. J Natl Compr Canc Netw 2014;12:727-733.

11. Schulman $C$, Cornel $E$, Matveev V, et al. Intermittent versus continuous androgen deprivation therapy in patients with relapsing or locally advanced prostate cancer: a phase $3 \mathrm{~b}$ randomised study (ICELAND). Eur Urol 2016;69:720-727.

12. Botrel TE, Clark $\mathrm{O}$, dos Reis $\mathrm{RB}$, et al. Intermittent versus continuous androgen deprivation for locally advanced, recurrent or metastatic prostate cancer: a systematic review and meta-analysis. BMC Urol 2014;14:9.

13. Dong $\mathrm{Z}$, Wang $\mathrm{H}, \mathrm{Xu} \mathrm{M}$, et al. Intermittent hormone therapy versus continuous hormone therapy for locally advanced prostate cancer: a meta-analysis. Aging Male 2015;18:233-237.

14. Hussain $M$, Tangen $C M$, Berry $D L$, et al. Intermittent versus continuous androgen deprivation in prostate cancer. N Engl J Med 2013;368:1314-1325.

15. Kolinsky M, Rescigno P, de Bono JS. Chemical or surgical castration-is this still an important question? JAMA Oncol 2016;2:437-438.

16. Sweeney CJ, Chen YH, Carducci M, et al. Chemohormonal therapy in metastatic hormone-sensitive prostate cancer. N Engl J Med 2015;373:737-746.

17. James ND, Sydes MR, Clarke NW, et al. Addition of docetaxel, zoledronic acid, or both to first-line long-term hormone therapy in prostate cancer (STAMPEDE): survival results from an adaptive, multiarm, multistage, platform randomised controlled trial. Lancet 2016;387:1163-1177.

18. Vale CL, Burdett S, Rydzewska LH, et al. Addition of docetaxel or bisphosphonates to standard of care in men with localised or metastatic, hormone-sensitive prostate cancer: a systematic review and meta-analyses of aggregate data. Lancet Oncol 2016;17:243-256. 